PROCEEDINGS OF THE

AMERICAN MATHEMATICAL SOCIETY

Volume 128, Number 4, Pages 1105-1108

S 0002-9939(99)05093-5

Article electronically published on August 5, 1999

\title{
SIGNED QUASI-MEASURES AND DIMENSION THEORY
}

\author{
D. J. GRUBB
}

(Communicated by Dale Alspach)

\begin{abstract}
A quasi-linear functional on $C(X)$ is a real-valued function that is linear on each closed, singly generated subalgebra and is norm bounded. We show that if the covering dimension $\operatorname{dim} X \leq 1$, then every quasi-linear functional on $C(X)$ is, in fact, linear. We do this by considering an associated set function, called a quasi-measure, and ask when such a set function can be extended to be a measure.
\end{abstract}

Let $X$ be a compact, Hausdorff space and $C(X)$ be the collection of all realvalued continuous functions on $X$. Let $\mathcal{A}(X)$ denote the collection of subsets of $X$ that are either open or closed. Also, for $f \in C(X)$, let $A(f)=\{\varphi \circ f: \varphi$ continuous on $f(X)\}$ be the closed subalgebra of $C(X)$ generated by $f$. A map $\rho: C(X) \rightarrow \mathbf{R}$ is called a quasi-linear functional if

a) $\rho$ is linear on each $A(f)$;

b) there is an $M<\infty$ such that $|\rho(f)| \leq M \cdot\|f\|_{\infty}$, for all $f \in C(X)$.

In [3], it was shown that, to every quasi-linear functional on $C(X)$, there is a real-valued set function $\mu: \mathcal{A}(X) \rightarrow \mathbf{R}$ with the following properties:

1. $\mu(\emptyset)=0$.

2. If $A, B \in \mathcal{A}(X)$ are disjoint and $A \cup B \in \mathcal{A}(X)$, then $\mu(A \cup B)=\mu(A)+\mu(B)$.

3. There is a constant $M<\infty$ such that whenever $\left\{A_{\alpha}\right\}$ is a disjoint collection of sets in $\mathcal{A}(X)$, then $\sum\left|\mu\left(A_{\alpha}\right)\right| \leq M$.

4. If $U$ is open and $\epsilon>0$, there is a closed set $K \subseteq U$ such that whenever $V$ is open and $V \subseteq U \backslash K$, then $|\mu(V)|<\epsilon$.

Such a set function is called a signed quasi-measure. For a signed quasi-measure, $\mu$, the corresponding quasi-linear functional is constructed as follows. For each $f \in C(X)$, there is a signed measure $\mu_{f}$ on $\mathbf{R}$ such that $\mu_{f}(U)=\mu\left(f^{-1} U\right)$. Then $\rho(f)$ is the integral of $i(x)=x$ with respect to $\mu_{f}$. See [3] for details about this correspondence and basic facts about signed quasi-measures.

The fact that a quasi-measure can exist that does not extend to a measure is not obvious. The first construction of such a quasi-measure is in [1] for the space $X=[0,1]^{2}$. However, for the case of positive quasi-measures, it was shown by Wheeler [8] and Shakmatov [7] that every quasi-measure is a measure if the covering dimension $\operatorname{dim} X \leq 1$. See also [4]. It is the goal of this paper to prove the analogous result for signed quasi-measures. This is not automatic from the result of Wheeler and Shakmatov since it is not known whether every signed quasi-measure

Received by the editors February 10, 1998 and, in revised form, June 1, 1998.

1991 Mathematics Subject Classification. Primary 28C15.

(C)2000 American Mathematical Society 
is a difference of two positive quasi-measures. The techniques of this paper shed no light on this question.

Our main result will be proved with the help of an extension theorem that is of interest in its own right.

Theorem 1. Let $\mu$ be a signed quasi-measure on $X$ and assume that whenever $U, V \subseteq X$ are open we have $\mu(U)+\mu(V)=\mu(U \cup V)+\mu(U \cap V)$. Then $\mu$ extends to a regular Borel signed measure on $X$.

Proof. Suppose $\mu$ satisfies the given condition. We first extend $\mu$ to sets of the form $U_{1} \backslash U_{2}$ where $U_{1}$ and $U_{2}$ are open via the definition $\mu\left(U_{1} \backslash U_{2}\right)=\mu\left(U_{1}\right)-$ $\mu\left(U_{1} \cap U_{2}\right)=\mu\left(U_{1} \cup U_{2}\right)-\mu\left(U_{2}\right)$. We first show this is well defined. This is actually a special case of Theorem 3.5.1(iii) of [6], however we include the proof for the sake of completeness. If $U_{1} \backslash U_{2}=V_{1} \backslash V_{2}$, then $U_{1} \cup U_{2} \cup V_{2}=V_{1} \cup V_{2} \cup U_{2}$ and $\left(U_{1} \cup U_{2}\right) \cap V_{2}=\left(V_{1} \cup V_{2}\right) \cap U_{2}$ so

$$
\begin{aligned}
\mu\left(U_{1} \cup U_{2}\right)+\mu\left(V_{2}\right) & =\mu\left(U_{1} \cup U_{2} \cup V_{2}\right)+\mu\left(\left(U_{1} \cup U_{2}\right) \cap V_{2}\right) \\
& =\mu\left(V_{1} \cup V_{2} \cup U_{2}\right)+\mu\left(\left(V_{1} \cup V_{2}\right) \cap U_{2}\right) \\
& =\mu\left(V_{1} \cup V_{2}\right)+\mu\left(U_{2}\right) .
\end{aligned}
$$

Thus $\mu\left(U_{1} \cup U_{2}\right)-\mu\left(U_{2}\right)=\mu\left(V_{1} \cup V_{2}\right)-\mu\left(V_{2}\right)$, as desired.

It is now a routine exercise to show that if $A=U \backslash V$ with $U$ and $V$ open, then $\mu(A)=\lim _{K \subseteq A} \mu(K)$ where $K$ ranges over closed subsets of $A$. This simply uses the regularity assumed for $\mu$.

Now we notice that, in general,

$$
\begin{aligned}
\mu((U \backslash V) \cap W)+\mu((U \backslash V) \backslash W) \\
\quad=\mu(U \cap W)-\mu(U \cap V \cap W)+\mu(U)-\mu(U \cap(V \cup W)) \\
\quad=\mu(U \cap W)+\mu(U)-\mu((U \cap V) \cap(U \cap W))-\mu((U \cap V) \cup(U \cap W)) \\
\quad=\mu(U \cap W)+\mu(U)-\mu(U \cap V)-\mu(U \cap W) \\
\quad=\mu(U \backslash V) .
\end{aligned}
$$

We recall the definition of the total variation of a quasi-measure as introduced in [3]. If $A$ is a set which is either open or closed,

$$
|\mu|(A)=\sup \left\{\sum_{1}^{n}\left|\mu\left(K_{i}\right)\right|: \bigsqcup_{1}^{n} K_{i} \subseteq A, K_{i} \text { closed }\right\} .
$$

Here we use the notation $\bigsqcup_{1}^{n} K_{i}$ to denote a disjoint union. By regularity of $\mu$, we have, under the conditions of the theorem,

$$
|\mu|(A)=\sup \left\{\sum_{1}^{n}\left|\mu\left(U_{i} \backslash V_{i}\right)\right|: \bigsqcup_{1}^{n}\left(U_{i} \backslash V_{i}\right) \subseteq A, U_{i}, V_{i} \text { open }\right\} .
$$

It is clear that

a) $|\mu|(\emptyset)=0$.

b) $U \subseteq V$ open implies $|\mu|(U) \leq|\mu|(V)$.

c) If $U$ is open and $\epsilon>0$, there is a closed set $K \subseteq U$ with $|\mu|(U \backslash K)<\epsilon$.

We also show

d) If $K$ is closed and $K \subseteq U$ open, then

$$
|\mu|(U)=|\mu|(K)+|\mu|(U \backslash K) .
$$


In fact, if $\bigsqcup_{1}^{n} K_{i} \subseteq U$ are closed, then

$$
\begin{aligned}
\sum\left|\mu\left(K_{i}\right)\right| & \leq \sum\left|\mu\left(K_{i} \backslash(X \backslash K)\right)\right|+\sum\left|\mu\left(K_{i} \cap(X \backslash K)\right)\right| \\
& \leq|\mu|(K)+|\mu|(U \backslash K) .
\end{aligned}
$$

Also,

e) $U, V$ open implies $|\mu|(U \cup V) \leq|\mu|(U)+|\mu|(V)$.

In fact, if $\bigsqcup_{1}^{n}\left(U_{i} \backslash V_{i}\right) \subseteq U \cup V$, then

$$
\begin{aligned}
\sum\left|\mu\left(U_{i} \backslash V_{i}\right)\right| & \leq \sum\left|\mu\left(\left(U_{i} \backslash V_{i}\right) \cap U\right)\right|+\sum \mid \mu\left(\left(U_{i} \backslash V_{i}\right) \backslash U\right) \\
& \leq|\mu|(U)+|\mu|(V) .
\end{aligned}
$$

Thus $|\mu|$ is a positive quasi-measure which is subadditive on open sets. By a theorem of Wheeler [8], $|\mu|$ extends to a regular Borel measure on $X$. If we now set $\nu=|\mu|-\mu$, then $\nu$ is a positive quasi-measure which satisfies the modularity property on open sets. Thus $\nu$ is also subadditive and hence extends to a regular Borel measure on $X$. Since $\mu=|\mu|-\nu, \mu$ also extends to a regular Borel signed measure.

It should be noted at this point that, for a general quasi-measure, $|\mu|$ need not be a quasi-measure. See 3 for an example.

Next we give a condition guaranteeing the modularity assumption of the theorem.

Lemma 2. Let $\mu$ be a signed quasi-measure and $U, V \subseteq X$ open sets with $\partial U \cap$ $\partial V=\emptyset$. Then

$$
\mu(U \cup V)+\mu(U \cap V)=\mu(U)+\mu(V) .
$$

Proof. In fact $U=(U \cap V) \cup(U \backslash \bar{V}) \cup(U \cap \partial V)$. Since $U \cap \partial V=\bar{U} \cap \partial V$ is closed, and this is a disjoint union,

$$
\mu(U)=\mu(U \cap V)+\mu(U \backslash \bar{V})+\mu(U \cap \partial V) .
$$

Similarly,

$$
\mu(V)=\mu(U \cap V)+\mu(V \backslash \bar{U})+\mu(V \cap \partial U) .
$$

Since all the sets involved in these expressions are pairwise disjoint, the result follows.

Now we can give the main theorem.

Theorem 3. Let the covering dimension $\operatorname{dim} X \leq 1$. Then every signed quasimeasure extends to a regular Borel signed measure.

Proof. Let $U, V \subseteq X$ be open and $\epsilon>0$. Let $K \subseteq U$ and $L \subseteq V$ be any closed sets. Since $\operatorname{dim} X \leq 1$, by Proposition 3.1.7 of [5], there are open sets $V_{1}$ and $V_{2}$ such that $K \subseteq V_{1} \subseteq U, L \subseteq V_{2} \subseteq V$ and $\partial V_{1} \cap \partial V_{2}=\emptyset$.

Since $\mu\left(V_{1} \cup V_{2}\right)+\mu\left(V_{1} \cap V_{2}\right)=\mu\left(V_{1}\right)+\mu\left(V_{2}\right)$ by the lemma, inner regularity of $\mu$ gives $\mu(U \cup V)+\mu(U \cap V)=\mu(U)+\mu(V)$. Now the first theorem gives the result.

Since there are positive quasi-measures on the unit square that do not extend to measures, this theorem gives the best possible result along these lines. It is not known whether the converse to this theorem is true. 
Corollary 4. If $\operatorname{dim} X \leq 1$, then every quasi-linear functional on $C(X)$ is linear.

This follows from the correspondence between quasi-measures and quasi-linear functionals.

Now recall that the analytic subalgebra generated by $f \in C(X)$ is the collection of $g \in C(X)$ which are constant on components of level sets of $f$. For example, $C\left(S^{1}\right)$ is singly generated analytically, but not as a subalgebra. More information about analytic subalgebras may be found, for example in [2]. In particular, we make essential use of a result of Katetov relating $\operatorname{dim} X$ and the number of analytic generators of $C(X)$.

Theorem 5. Any quasi-linear map is linear on singly generated analytic subalgebras of $C(X)$.

Proof. Let $\rho: C(X) \rightarrow \mathbf{R}$ be a quasi-linear map and let $f \in C(X)$. Consider the space $Y$ obtained from $X$ by identifying components of level sets of $f$ to points. Let $q: X \rightarrow Y$ be the quotient map. Then $\eta(g)=\rho(g \circ q)$ defines a quasi-linear functional on $C(Y)$. However, the space $Y$ obtained in this way has $\operatorname{dim} Y \leq 1$ since $C(Y)$ is singly generated analytically. Thus $\eta$ is linear. Since the analytic subalgebra generated by $f$ is exactly the collection of functions of the form $g \circ q$, we are done.

\section{REFERENCES}

[1] Johan Aarnes, Quasi-states and quasi-measures, Adv. in Math. 86, (1991) pp. 41-67. MR 92d:46152

[2] L. Gillman and M. Jerison, Rings of Continuous Functions, Springer-Verlag, New York, 1960. MR 22:6994

[3] D. J. Grubb, Signed Quasi-Measures, Trans. Amer. Math. Soc. 349, (1997) pp. 1081-1089. MR 98c:28012

[4] D. J. Grubb and Tim LaBerge, Spaces of quasi-measures, accepted Bull. Math. Canad.

[5] A. R. Pears, Dimension Theory of General Spaces, Cambridge University Press, London 1975. MR 52:15405

[6] K. P. S. Rao and M. B. Rao, Theory of Charges, Academic Press, New York, 1983. MR 86f:28006

[7] Dmitri Shakmatov, Linearity of quasi-states on commutative $C^{*}$ algebras of stable rank 1. unpublished.

[8] Robert Wheeler, Quasi-Measures and Dimension Theory, Top. Appl. 66, (1995) pp. 75-92. MR 96m:28002

Department of Mathematical Sciences, Northern Illinois University, De Kalb, IlliNOIS 60115

E-mail address: grubb@math.niu.edu 